\title{
Transversality for Critical Relations of Families of Rational Maps: An Elementary Proof
}

\author{
Genadi Levin, Weixiao Shen and Sebastian van Strien
}

In memory of our dear friend and colleague Welington de Melo.

\begin{abstract}
In this paper we will give a short and elementary proof that critical relations unfold transversally in the space of rational maps.
\end{abstract}

Keywords Holomorphic dynamics · Rational maps • Transversality

\section{Introduction}

In this short paper we will give an elementary proof of some transversality properties for families of rational maps. We will consider the space $\operatorname{Rat}_{d}^{\mu}$ of rational maps of degree $d$ with precisely $v$ critical points of multiplicities $\left(\mu_{1}, \mu_{2}, \ldots, \mu_{v}\right)$. In Theorem 2.1 we will show that this space of maps can be locally parametrised by critical values. Given $f \in \mathbf{R a t}_{d}^{\mu}$, let $\zeta=\zeta(f) \geq 0$ be the maximal number of critical points with pairwise disjoint infinite orbits and define $N=v-\zeta(f)$. In Theorem 3.2 we will show that if $f$ is not a flexible Lattès map then one can organise the set of critical relations of $f$ in the form

G. Levin

Hebrew University, Jerusalem, Israel

e-mail: genady.levin@mail.huji.ac.il

W. Shen

Shanghai Center for Mathematical Sciences, Fudan University, Shanghai, China

e-mail:wxshen@fudan.edu.cn

S. van Strien $(\otimes)$

Imperial College, London, UK

e-mail: s.van-strien@imperial.ac.uk

(C) The Author(s) 2019 


$$
\left\{f^{m_{k}}\left(c_{i_{k}}\right)=f^{n_{k}}\left(c_{j_{k}}\right), k=1, \ldots, N\right\}
$$

so that the map

$$
\boldsymbol{R a t}_{d}^{\mu} \ni g \mapsto\left\{\sigma\left(g^{m_{k}}\left(c_{i_{k}}(g)\right)\right)-\sigma\left(g^{n_{k}}\left(c_{j_{k}}(g)\right)\right)\right\}_{k=1}^{N}
$$

has maximal rank for $g$ near $f$, where $\sigma$ is any Möbius transformation with $\sigma\left(f^{m_{k}}\left(c_{i_{k}}\right)\right) \neq \infty$. Property (1.1) obviously is a transversality condition.

In fact, the choice of critical relations is in general not unique, but as long as the selected collection is full, as made explicit in Definition 3.6 below, the maximal rank property holds.

Indeed, we should emphasise that some care is required in the choice of critical relations. For example, in the case of $f_{t}(z)=z^{2}+t$ with $t=0$, the derivative of $t \mapsto f_{t}^{2}(0)-f_{t}(0)$ vanishes at $t=0$. The correct way of expressing transversal unfolding of the critical relation $f_{t}(0)=0$ in (1.1) is by taking $m_{1}=1$ and $n_{1}=0$ in this equation, i.e. by asserting that the derivative $t \mapsto f_{t}(0)-0$ is non-zero at $t=0$.

In the unicritical case, transversal unfolding of critical relations in the pre-periodic case goes back to Douady and Hubbard [5] and Tsujii [32], see also [16, Remark 5.10]. Milnor and Thurston [28] and Sullivan, see [25, Theorem VI.4.2], proved a 'topological' version of transversality.

An abstract approach to transversality for finite type maps was developed by A. Epstein, see [8, 9], obtaining in Part 1 of [8] transversality within the Teichmüller deformation space $\operatorname{Def}_{A}^{B}(f)$, and in Sect. 5.4 in [8] the loci defined by critical relations within $\operatorname{Def}_{A}^{B}(f)$ is discussed. Part 2, and in particular Sect.10, of [8] goes into a strategy for transferring the transversality results obtained in $\operatorname{Def}_{A}^{B}(f)$ to the space of rational functions. However, we were not able to find an explicit statement covering Theorem 3.2 or Theorem 3.3. Nevertheless, it is likely that the strategy in [8] can be executed to obtain statements similar to the ones in this paper.

Our results also hold in the setting of degenerate critical points and gives unfoldings of critical relations even when critical points share the same critical value. For this we use that $\mathbf{R a t}_{d}^{\mu}$ is a manifold and that $\mathbf{R a t}_{d}^{\boldsymbol{\mu}} \ni f \mapsto\left(f\left(c_{1}\right), \ldots, f\left(c_{v}\right)\right)$ has rank $v$, see Theorem 2.1 .

In this short and self-contained paper we prove transversality following the approach developed by Levin in [17-20], see also [15]. The starting point of this paper are calculations from $[18,20]$ which show that if the transversality property (1.1) fails at $g=f$, then one can construct a non-zero integrable meromorphic quadratic differential that is invariant under push-forward by $f$, which in turn implies that $f$ is a flexible Lattès example. Indeed, the main Theorem 3.3 can be proved as in [20], see Remark 5.1, although we shall provide a more direct and shorter proof in this paper.

The idea of using quadratic differentials appeared first in Thurston's characterization of post-critically finite branched covering of the 2-sphere [6]. It has been used in for example [7, 15] and this was also used in [33] to obtain a similar statement to ours for the quadratic case. 
Theorem 3.2 was proved previously for the case that critical points are nondegenerate and eventually mapped into repelling periodic orbits, but never into a critical point, see [3, 30] and also [13, Theorem 4.8].

Transversality also holds in other settings. For example, if each critical point is mapped into a hyperbolic set, see [30], when a summability condition holds along the orbit of critical values, see [2,20], for the unfolding of multipliers of periodic orbits, see $[8,18]$ and for a large class of interval maps, see [21].

As mentioned, the aim of this paper is to present a proof of transversality for rational maps with critical relations in a complete and readily accessible form.

In Sect. 6 we discuss corresponding results for polynomials.

\section{Parametrising Rational Maps by Their Critical Values}

Let $\mathbf{R a t}_{d}$ denote the collection of all rational maps of degree $d \geq 2$. This space is naturally parameterized by an open set in $P \mathbb{C}^{2 d+1}$.

Given a non-ordered list $\boldsymbol{\mu}=\left(\mu_{1}, \mu_{2}, \ldots, \mu_{\nu}\right)$ with $\sum_{i=1}^{v} \mu_{i}=2 d-2$, we say a rational map $f \in \mathbf{R a t}_{d}$ is in the class $\mathbf{R a t}_{d}^{\mu}$ if $f$ has precisely $v$ distinct critical points $c_{1}, c_{2}, \ldots, c_{\nu}$ with multiplicities $\mu_{1}, \mu_{2}, \ldots, \mu_{\nu}$ respectively. Taking $\mathbf{1}=(1, \ldots, 1)$, $\operatorname{Rat}_{d}{ }_{d}$ corresponds to the space of rational maps with $2 d-2$ non-degenerate critical points.

Rational maps are not fully determined by their critical values (not even on small open subsets $W \subset \mathbf{R a t}_{d}^{\mu}$ ), because one can precompose a rational map by a Möbius transformation without changing its critical values. However one can find a neighbourhood $W$ of $f$ and a normalisation (based on precompositions with Möbius transformations) so that critical values parametrise all maps in $W$ satisfying this normalisation:

Theorem 2.1 For each $\boldsymbol{\mu}, \boldsymbol{R a t}_{d}^{\mu}$ is an embedded submanifold of dimension $v+3$ of $\boldsymbol{R a t}_{d}$ and the functions defined by the critical values form a partial holomorphic local coordinate system, i.e. the map $\boldsymbol{R a t}_{d}^{\mu} \ni f \mapsto\left(f\left(c_{1}\right), \ldots, f\left(c_{v}\right)\right)$ has rank $v$ and can be completed by 3 other coordinates to be a holomorphic coordinate system.

Remark 2.1 Theorem 2.1 is not new. Similar statements are proved e.g. in [11, 19] (see also [12]) using the Measurable Riemann Mapping Theorem with dependence on parameters; the idea of those proofs goes back probably to [31]. Our proof borrows an idea of Douady and Sentenac [29, Appendix A], and is short and elementary. The case $\mu_{\nu}=d-1$ corresponds to the polynomial case, which in some real cases was dealt with in [25, p. 120] and [29], see also [10].

Theorem 2.1 follows from Proposition 2.2 below. Assume without loss of generality (by post and pre composing $f$ by Möbius transformations if necessary) that the critical points and the critical values avoid the point at $\infty$. Then for each $i=1,2, \ldots, v$, 


$$
f^{\prime}\left(c_{i}\right)=f^{\prime \prime}\left(c_{i}\right)=\cdots=f^{\left(\mu_{i}\right)}\left(c_{i}\right)=0, f^{\left(\mu_{i}+1\right)}\left(c_{i}\right) \neq 0 .
$$

Applying the Implicit Function Theorem to the maps $\left(g, \zeta_{i}\right) \mapsto g^{\left(\mu_{i}\right)}\left(\zeta_{i}\right)$ for $\left(g, \zeta_{i}\right)$ near $\left(f, c_{i}\right)$, gives that there exists a neighborhood $W$ of $f$ in $\mathbf{R a t}_{d}$ and uniquely defined functions $\zeta_{i}: W \rightarrow \mathbb{C}$ which are holomorphic such that $\zeta_{i}(f)=c_{i}$ and $g^{\left(\mu_{i}\right)}\left(\zeta_{i}(g)\right)=0, g^{\left(\mu_{i}+1\right)}\left(\zeta_{i}(g)\right) \neq 0$ for each $g \in W$. Replacing $W$ by a smaller neighborhood, for each $g \in W$ the equation $g^{\prime}(\zeta)=0$ has $\mu_{i}$ solutions $\zeta$ (counting multiplicity) near $c_{i}$. It follows that for any $g \in W \cap \mathbf{R a t}_{d}^{\mu}, \quad g^{\prime}(\zeta)=0$ has a unique solution near $c_{i}$ (with multiplicity $\mu_{i}$ ); hence $\zeta_{i}(g)$ is the only critical point of $g \in W \cap \boldsymbol{R a t}_{d}^{\mu}$ near $c_{i}$ and it has multiplicity $\mu_{i}$.

For $g \in W$, write

$$
\zeta_{i}^{0}(g)=g\left(\zeta_{i}(g)\right), \zeta_{i}^{1}(g)=g^{\prime}\left(\zeta_{i}(g)\right), \zeta_{i}^{2}(g)=g^{\prime \prime}\left(\zeta_{i}(g)\right), \ldots
$$

Thus $\zeta_{i}(g)$ is a critical point of $g$ with multiplicity $\mu_{i}$ if and only if $\zeta_{i}^{j}(g)=0$ for all $1 \leq j \leq \mu_{i}-1$ (note that $g^{\left(\mu_{i}\right)}\left(\zeta_{i}(g)\right)=0, g^{\left(\mu_{i}+1\right)}\left(\zeta_{i}(g)\right) \neq 0$ holds automatically for all $g \in W$ ). Define $G: W \rightarrow \mathbb{C}^{2 d-2}$ by

$$
g \rightarrow\left(\zeta_{1}^{0}(g), \zeta_{1}^{1}(g), \ldots, \zeta_{1}^{\left(\mu_{1}-1\right)}(g), \ldots, \zeta_{v}^{0}(g), \zeta_{v}^{1}(g), \ldots, \zeta_{v}^{\left(\mu_{\nu}-1\right)}(g)\right) .
$$

Since $W$ has dimension $2 d+1$, Theorem 2.1 follows immediately from:

Proposition 2.2 For each rational map $f$ as above, the Jacobian of $G$ has rank $2 d-2$ at $g=f$.

This proposition also immediately implies:

Corollary 2.3 Assume that all critical points of $f$ are non-degenerate. Then there exists a neighbourhood $W$ of $f$ in $\boldsymbol{R a t}_{d}$ so that the critical points $c_{1}(g), \ldots, c_{2 d-2}(g)$ of $g$ depend holomorphically on $g \in W$ and the Jacobian of the map

$$
g \mapsto\left(g\left(c_{1}(g)\right), g\left(c_{2}(g)\right), \ldots, g\left(c_{2 d-2}(g)\right)\right)
$$

has maximal rank at every $g \in W$.

\subsection{Proof of Proposition 2.2}

Proof of Proposition 2.2. Arguing by contradiction, assume that the assertion of the proposition is false. Then there exist complex numbers $A_{i}^{j}, 1 \leq i \leq \nu, 0 \leq j<\mu_{i}$, not all equal to zero, such that all partial derivatives of the map

$$
\mathbf{G}(g)=\sum_{i=0}^{\nu} \sum_{j=0}^{\mu_{i}-1} A_{i}^{j} \zeta_{i}^{(j)}(g)
$$


are equal to zero at $g=f$. This means that for any holomorphic curve $f_{t}$ in Rat $_{d}$, passing through $f$ at $t=0$, the map $G(t)=\mathbf{G}\left(f_{t}\right)$ satisfies $G^{\prime}(0)=0$. Let us write

$$
f_{t}(z)=\frac{\sum_{k=0}^{d} a_{k}(t) z^{k}}{\sum_{k=0}^{d} b_{k}(t) z^{k}}=: \frac{P_{t}(z)}{Q_{t}(z)},
$$

where $a_{k}(t), b_{k}(t)$ are holomorphic in a neighborhood of 0 and $P_{0}$ and $Q_{0}$ are coprime polynomials. For $1 \leq i \leq v, j=0, \ldots, \mu_{i}-1$ define $v_{i, j}(t)=\zeta_{i}^{(j)}\left(f_{t}\right)$. Then

$$
v_{i, j}^{\prime}(0)=\left.\left(\frac{\sum_{k=0}^{d} a_{k}^{\prime}(0) z^{k} Q_{0}(z)-\sum_{l=0}^{d} b_{l}^{\prime}(0) z^{l} P_{0}(z)}{Q_{0}(z)^{2}}\right)^{(j)}\right|_{z=c_{i}}
$$

where we use $f^{(j+1)}\left(\zeta_{i}(f)\right)=f^{(j+1)}\left(c_{i}\right)=0$. So

$$
\begin{aligned}
0=G^{\prime}(0) & =\sum_{i, j} A_{i}^{j} v_{i, j}^{\prime}(0) \\
& =\left.\sum_{i, j} A_{i}^{j}\left(\frac{\sum_{k=0}^{d} a_{k}^{\prime}(0) z^{k} Q_{0}(z)-\sum_{l=0}^{d} b_{l}^{\prime}(0) z^{l} P_{0}(z)}{Q_{0}(z)^{2}}\right)^{(j)}\right|_{z=c_{i}} .
\end{aligned}
$$

We claim that for any polynomial $T$, we have

$$
\left.\sum_{i, j} A_{i}^{j}\left(\frac{T(z)}{Q_{0}(z)^{2}}\right)^{(j)}\right|_{z=c_{i}}=0
$$

To see this, first notice that since $T_{0}(z)=\prod_{i=1}^{v}\left(z-c_{i}\right)^{\mu_{i}}$ has a zero at $z=c_{i}$ of multiplicity $\mu_{i}$, the Eq. (2.2) holds for $T=T_{0}$ and $T=T_{0} U$, where $U$ is an arbitrary polynomial. Since $\operatorname{deg}\left(T_{0}\right)=2 d-2$ and any polynomial can be written as $T_{0} U+T$ where $\operatorname{deg}(T)<2 d-2$ it therefore suffices to prove (2.2) in the case that $\operatorname{deg}(T)<$ $2 d-2$. For such a polynomial $T$, we can find polynomials $R, S$ of degree at most $d-1$ such that $T=R Q_{0}-S P_{0}$, since $P_{0}$ and $Q_{0}$ are coprime and one of them has degree $d$. Choosing $a_{k}, b_{l}$ suitably such that $R(z)=\sum_{k} a_{k}^{\prime}(0) z^{k}$ and $S=\sum_{l} b_{l}^{\prime}(0) z^{l}$ and applying (2.1), we obtain (2.2).

We shall now deduce from this equation that $A_{i}^{j}=0$ for all $i, j$ and thus obtain a contradiction. Indeed, (2.2) implies that for any polynomial $V$, we have

$$
\sum_{i, j} A_{i}^{j} V^{(j)}\left(c_{i}\right)=0 .
$$

Fix $1 \leq i_{0} \leq v, 1 \leq j_{0}<\mu_{i_{0}}$, take 


$$
V(z)=\prod_{i \neq i_{0}}\left(z-c_{i}\right)^{\mu_{i}}\left(z-c_{i_{0}}\right)^{j_{0}} .
$$

Then $V^{\left(j_{0}\right)}\left(c_{i_{0}}\right) \neq 0$ and $V^{(j)}\left(c_{i}\right)=0$ for any other $(i, j)$. Therefore $A_{i_{0}}^{j_{0}}=0$. The proof is completed.

\section{Transversality Results for Rational Maps}

Throughout this section we again consider a map $f$ in the space $\mathbf{R a t}_{d}^{\boldsymbol{\mu}}$ of rational maps of degree $d$, with $v$ distinct critical points $c_{1}, c_{2}, \ldots, c_{v}$ with multiplicities $\boldsymbol{\mu}=\left(\mu_{1}, \mu_{2}, \ldots, \mu_{v}\right)$ where $\sum_{i=1}^{v} \mu_{i}=2 d-2$. For $g$ in a small neighborhood of $f$ in $\mathbf{R a t}_{d}^{\mu}$, the critical points $c_{1}(g), c_{2}(g), \ldots, c_{v}(g)$ depends holomorphically on $g$.

We are interested in the smoothness of sets defined by a set of critical relations of the form $g^{m}\left(c_{i}(g)\right)=g^{n}\left(c_{j}(g)\right)$. A particular case of our main result in this direction is the following:

Theorem 3.1 Let $f \in \boldsymbol{R a t}_{d}^{\mu}$ and assume that there exists $1 \leq i, j \leq v$ and $m>0$ so that $f^{m}\left(c_{i}\right)=c_{j}$. Then the equation

$$
g^{m}\left(c_{i}(g)\right)=c_{j}(g)
$$

defines an embedded submanifold of $\boldsymbol{R a t}_{d}^{\mu}$ of codimension one near $f$.

In order to state a more general result, we have to prepare some terminology. Let us say that a quadruple $(i, j ; m, n)$ is a (candidate) critical relation if $1 \leq i, j \leq v$, and $m, n$ are non-negative integer with $m+n>0$. We say that this critical relation is realized by $f$ if $f^{m}\left(c_{i}(f)\right)=f^{n}\left(c_{j}(f)\right)$.

Given $f$, let $\zeta=\zeta(f) \geq 0$ be the maximal number of critical points with pairwise disjoint infinite orbits. Note that this number is well-defined, but that one cannot say which critical points are 'free'. For example, if $f$ has three distinct critical points $c_{1}, c_{2}, c_{3}$, so that the forward orbits of $f\left(c_{1}\right)=f\left(c_{2}\right)$ and $c_{3}$ are disjoint and infinite, then $\zeta(f)=2$; of course one could consider $c_{1}, c_{3}$ as the free critical points of $f$, but equally well also $c_{2}, c_{3}$.

In this section we will show

Theorem 3.2 Assume $f \in \boldsymbol{R a t}_{d}^{\boldsymbol{\mu}}$ is not a flexible Lattès map. Then there exists a set

$$
\mathcal{F}=\left\{\left(i_{k}, j_{k} ; m_{k}, n_{k}\right), k=1, \ldots, N\right\} \text { with } N=v-\zeta(f)
$$

of critical relations $f^{m_{k}}\left(c_{i_{k}}\right)=f^{n_{k}}\left(c_{j_{k}}\right)$ which are realised by $f$, such that the Jacobian of the map

$$
\mathcal{R}_{\mathcal{F}}^{\sigma}: g \mapsto\left(\sigma\left(g^{m_{k}}\left(c_{i_{k}}(g)\right)\right)-\sigma\left(g^{n_{k}}\left(c_{j_{k}}(g)\right)\right)\right)_{k=1}^{N}
$$


at $g=f$ has rank $N$, whenever $\sigma$ is a Möbius transformationfor which $\sigma\left(f^{m_{k}}\left(c_{i_{k}}\right)\right) \in$ $\mathbb{C}, k=1, \ldots, N$.

Remark 3.1 The assumption that $\sigma\left(f^{m_{k}}\left(c_{i_{k}}\right)\right) \in \mathbb{C}$ is made to ensure that (3.1) is holomorphic near $f$. The kernel of the Jacobian of $\mathcal{R}_{\mathcal{F}}^{\sigma}$ at $f$, hence its rank, does not depend on $\sigma$, as long as $\sigma\left(f^{m_{k}}\left(c_{i_{k}}\right)\right) \neq \infty$ for all $k=1, \ldots, N$. Indeed, a tangent vector of $\mathbf{R a t}_{d}^{\mu}$ at $f$ belongs to the kernel if and only if it has the same image under the tangent map of the maps $g \mapsto\left(g^{m_{k}}\left(c_{i_{k}}(g)\right)\right)_{k=1}^{N}$ and $g \mapsto\left(g^{n_{k}}\left(c_{j_{k}}(g)\right)\right)_{k=1}^{N}$ at $g=f$ (both are holomorphic maps from a neighborhood of $f$ in $\mathbf{R a t}_{d}^{\mu}$ into $\overline{\mathbb{C}}^{N}$ ).

In particular, to prove Theorem 3.2, we can and will assume that the critical obits of $f$ avoid $\infty$ and only prove that $\mathcal{R}_{\mathcal{F}}=\mathcal{R}_{\mathcal{F}}^{i d}$ has rank $N$ at $g=f$. Indeed, we can always choose $z_{0}$ (arbitrarily close to $\infty$ ) which avoids the critical orbits of $f$. Put $\sigma(z)=z_{0} z /\left(z_{0}-z\right)$ and $\tilde{f}=\sigma \circ f \circ \sigma^{-1}$. Then $\infty$ avoids the critical orbits of $\tilde{f}$. Since $\mathcal{R}_{\mathcal{F}}^{\sigma}(g)=\mathcal{R}_{\mathcal{F}}^{i d}\left(\sigma \circ g \circ \sigma^{-1}\right)$, once we prove that the Jacobian of $g \mapsto \mathcal{R}_{\mathcal{F}}^{i d}(g)$ has rank $N$ at $g=\tilde{f}$, it follows that the Jacobian of $\mathcal{R}_{\mathcal{F}}^{\sigma}$ has rank $N$ at $g=f$.

Remark 3.2 There are several ways of assigning a set of critical relations $\mathcal{F}$ to $f$. As we will prove in Sect. 4.2, for any set of critical relations which is full in the sense of Definition 3.6, Theorem 3.2 holds.

Remark 3.3 A flexible Lattès map is by definition a rational map that is conformally conjugate to a map of the form $L / \sim: T / \sim T / \sim$, where $T=\mathbb{C} /(\mathbb{Z} \oplus \gamma \mathbb{Z}), \gamma \in \mathbb{H}$ (where $\mathbb{H}$ is the upper-half plane), $\sim$ is the equivalence relation on $\mathbb{C}$ defined by $z \sim-z$ and $L: \mathbb{C} \rightarrow \mathbb{C}$ is of the form $L(z)=a z+b$ with $a \in \mathbb{Z}$ and $2 b \in \mathbb{Z} \oplus \gamma \mathbb{Z}$, see [27]. Such maps can be of two types: either each critical point is mapped in two iterates into a repelling fixed point or in one iterate into a repelling periodic point of period two, see [27].

Remark 3.4 Theorem 3.2 and the implicit function theorem, imply that manifolds defined by critical relations corresponding to disjoint subsets $\mathcal{F}^{\prime}, \mathcal{F}^{\prime \prime}$ of $\mathcal{F}$ are smooth and transversal to one another.

For completeness we prove the following corollary of Theorem 3.2:

Corollary 3.5 If each critical point $c_{i}$ is eventually mapped to a repelling periodic point $p_{i}$ with $f^{m_{i}}\left(c_{i}\right)=p_{i}$ and $f^{j}\left(c_{i}\right) \notin\left\{c_{1}, \ldots, c_{v}\right\}$ for all $j=1, \ldots, m_{i}$ then the Jacobian of

$$
\boldsymbol{R a t}_{d}^{\mu} \ni g \mapsto\left\{\sigma\left(g^{m_{i}}\left(c_{i}(g)\right)\right)-\sigma\left(p_{i}(g)\right)\right\}_{i=1}^{v}
$$

has maximal rank at $g=f$, where $\sigma$ is a Möbius transformation with $\sigma\left(p_{i}\right) \neq \infty$ for all $i$.

Proof For the same reason as explained in Remark 3.1, we only need to consider the case where $\infty$ avoids the critical orbits and $\sigma=i d$. Let $\mathcal{R}$ denote the map in (3.2). The corollary follows from the following claim by Theorem 3.2.

Claim. If $f_{t}$ is a holomorphic curve in $\mathbf{R a t}_{d}^{\mu}$ passing through $f$ at $t=0$ which represents a vector in the kernel of $D_{f} \mathcal{R}$, then for any critical relations $(i, j ; m, n)$ realized by $f$, we have 


$$
f_{t}^{m}\left(c_{i}\left(f_{t}\right)\right)-f_{t}^{n}\left(c_{j}\left(f_{t}\right)\right)=o(t) \text { as } t \rightarrow 0
$$

Indeed, the claim implies that the kernel of $D_{f} \mathcal{R}$ is contained in the kernel of $D_{f} \mathcal{R}_{\mathcal{F}}$ for any finite collection $\mathcal{F}$ of critical relations. By Theorem 3.2, we can choose $\mathcal{F}$ such that $D_{f} \mathcal{R}_{\mathcal{F}}$ has maximal rank. Thus $D_{f} \mathcal{R}$ has maximal rank.

Let us prove the claim. Choose $k$ large enough such that $m+k \geq m_{i}$ and $n+k \geq$ $m_{j}$. Since $f^{m+k-m_{i}}\left(p_{i}\right)=f^{n+k-m_{j}}\left(p_{j}\right)$, the periodic points $p_{i}$ and $p_{j}$ have the same period, denoted by $s$. Moreover, if $p_{i}(t)$ (resp. $\left.p_{j}(t)\right)$ denotes the repelling periodic point of $f_{t}^{s}$ near $p_{i}$ (resp. $p_{j}$ ), then

$$
f_{t}^{m+k-m_{i}}\left(p_{i}(t)\right)=f_{t}^{n+k-m_{j}}\left(p_{j}(t)\right) .
$$

Since $f_{t}^{m_{i}}\left(c_{i}\left(f_{t}\right)\right)-p_{i}(t)=o(t)$ as $t \rightarrow 0$, we have

$$
f_{t}^{m+k}\left(c_{i}\left(f_{t}\right)\right)-f_{t}^{m+k-m_{i}} p_{i}(t)=o(t) \text { as } t \rightarrow 0 .
$$

Similarly, we have

$$
f_{t}^{n+k}\left(c_{j}\left(f_{t}\right)\right)-f_{t}^{n+k-m_{j}}\left(p_{j}(t)\right)=o(t) \text { as } t \rightarrow 0 .
$$

Therefore,

$$
f_{t}^{m+k}\left(c_{i}\left(f_{t}\right)\right)-f_{t}^{n+k}\left(c_{j}\left(f_{t}\right)\right)=o(t) \text { as } t \rightarrow 0 .
$$

Since $f^{m+k^{\prime}}\left(c_{i}\right)$ is not critical for each $0 \leq k^{\prime}<k$, it follows that $f_{t}^{m}\left(c_{i}\left(f_{t}\right)\right)-$ $f_{t}^{n}\left(c_{j}\left(f_{t}\right)\right)=o(t)$ as $t \rightarrow 0$.

\subsection{How to Associate Critical Relations to a Rational Map}

There are several ways to record the (infinitely many) critical relations of a rational map. In this subsection we will show how one can associate these in an efficient way so that in particular no critical relation is counted twice.

As above, let $c_{1}, c_{2}, \ldots, c_{v}$ be the critical points of a rational map in the class $\mathbf{R a t}_{d}^{\mu}$. For an arbitrary collection $\mathcal{F}$ of critical relations realized by $f$, let $\sim_{\mathcal{F}}$ denote the smallest equivalence relation in the set $\Sigma:=\{(i, m): 1 \leq i \leq v, m \geq 0\}$ such that $(i, m+k) \sim_{\mathcal{F}}(j, n+k)$ for each $(i, j ; m, n) \in \mathcal{F}$ and each $k \geq 0$.

So $\sim_{\mathcal{F}}$ defines the set of critical relations that can be 'read off' from $\mathcal{F}$. So for example, if $v=4$ and $\mathcal{F}=\{(1,2 ; 1,1),(1,3 ; 1,1)\}$ then $(i, 1+k) \sim_{\mathcal{F}}(j, 1+k)$ for all $i, j \in\{1,2,3\}$ and all $k \geq 0$, but $(i, m) \nsim_{\mathcal{F}}(4, n)$ for $i \in\{1,2,3\}$ and all $m, n \geq 0$.

Roughly speaking, we say that a collection $\mathcal{F}$ of critical relations is full if it 'essentially' explains all critical relations of $f$ and $\mathcal{F}$ is minimally full if it does not contain redundant critical relations. More precisely, 

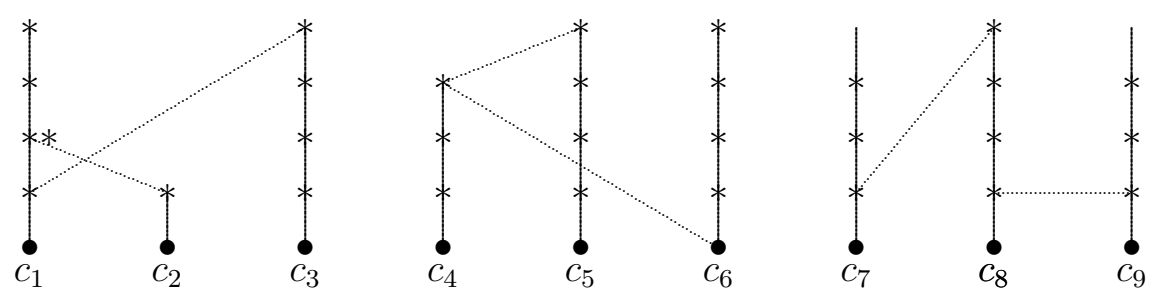

Fig. 1 The orbit diagram of a map with $\zeta(f)=3$ and $f^{2}\left(c_{1}\right)=f\left(c_{2}\right), f\left(c_{1}\right)=f^{4}\left(c_{3}\right), f^{3}\left(c_{4}\right)=$ $f^{4}\left(c_{5}\right)=c_{6}, f\left(c_{7}\right)=f^{4}\left(c_{8}\right), f\left(c_{8}\right)=f\left(c_{9}\right)$. Each of the collections $\{(2,1 ; 1,2),(3,1 ; 4,1)$, $(4,6 ; 3,0),(5,4 ; 4,3),(8,7 ; 4,1),(9,8 ; 1,1)\}, \quad\{(2,1 ; 1,2),(3,1 ; 4,1),(4,6 ; 3,0),(5,6 ; 4,0)$, $(9,7 ; 4,1),(9,8 ; 1,1)\}$ and $\{(2,1 ; 2,3),(3,1 ; 5,2),(4,6 ; 3,0),(5,6 ; 4,0),(9,7 ; 4,1),(9,8$; $2,2)\}$ is minimally full

Definition 3.6 We say that a collection $\mathcal{F}$ of critical relations realized by $f$ is full if for any critical relation $(i, j ; m, n)$ realised by $f$, i.e. whenever $f^{m}\left(c_{i}\right)=f^{n}\left(c_{j}\right)$, there exists $k \geq 0$ such that $(i, m+k) \sim_{\mathcal{F}}(j, n+k)$ and such that $f^{m+k^{\prime}}\left(c_{i}\right)=$ $f^{n+k^{\prime}}\left(c_{j}\right) \notin\left\{c_{1}, \ldots, c_{\nu}\right\}$ for each $0 \leq k^{\prime}<k$.

Note that any full collection contains at least $v-\zeta(f)$ relations. A full collection $\mathcal{F}$ is called minimally full if $\# \mathcal{F}=v-\zeta(f)$ (Fig. 1).

If $\mathcal{F}$ is minimally full then in particular there exists no $1 \leq i_{1}, i_{2}, \cdots, i_{k} \leq v$, $k \geq 2$, such that

$$
\left(i_{1}, i_{2} ; 1,1\right),\left(i_{2}, i_{3} ; 1,1\right), \ldots,\left(i_{k}, i_{1} ; 1,1\right) \in \mathcal{F}
$$

We refer to the last property as the non-cyclic condition.

So if $f$ has critical points $c_{1}, \ldots, c_{4}$ with critical relations $f^{k}\left(c_{1}\right)=f^{k}\left(c_{2}\right)=$ $f^{k}\left(c_{3}\right)$ for all $k \geq 1$ and there are no other critical relations, then $\zeta(f)=2$ and

$$
\mathcal{F}_{1}=\{(1,2 ; 1,1),(1,3 ; 1,1)\} \text { but also } \mathcal{F}_{2}=\{(1,2 ; 2,2),(1,3 ; 3,3)\}
$$

are minimally full collections.

Note that if $\mathcal{F}$ is minimally full and $(i, j ; m, n) \in \mathcal{F}$ then $(j, i ; n, m) \notin \mathcal{F}$. Later on, we will define a convenient choice for a minimally full collection $\mathcal{F}$, see Definition 4.2 and in Lemma 4.4 we will show that such a choice can always be made.

\subsection{An Even More General Theorem}

Let $g$ be a rational map with critical points $c_{1}(g), \ldots, c_{v}(g)$. Associate to each $(i, j ; m, n)$ the following rational map 


$$
Q_{i, j ; m, n}^{g}(z)=\sum_{r=1}^{m} \frac{D g^{m-r}\left(g^{r}\left(c_{i}(g)\right)\right)}{z-g^{r}\left(c_{i}(g)\right)}-\sum_{s=1}^{n} \frac{D g^{n-s}\left(g^{s}\left(c_{j}(g)\right)\right)}{z-g^{s}\left(c_{j}(g)\right)}
$$

when $g^{r}\left(c_{i}(g)\right), g^{s}\left(c_{j}(g)\right) \neq \infty$ for all $1 \leq r \leq m, 1 \leq s \leq n$. (Convention: For $m=$ 0 or $n=0$, the corresponding sum is understood as 0 .)

Given a meromorphic quadratic differential $Q=q(z) d z^{2}$, define its push-forward as $f_{*} Q=\widehat{q}(z) d z^{2}$, where

$$
\widehat{q}(z)=\sum_{w \in f^{-1}(z)} \frac{q(w)}{f^{\prime}(w)^{2}}
$$

It is not difficult to check that $f_{*} Q$ is again a meromorphic quadratic differential. The assignment $Q \mapsto f_{*} Q$ is often called the Thurston operator, see [14, 23], and was used in Thurston's rigidity theorem, see [6]. M. Tsujii was probably the first to use quadratic differentials in the context of transversality, see [26, 32, 33], but see also [2, 8, 9, 17, 18, 20, 22].

Theorem 3.2 will follow from

Theorem 3.3 Assume that the critical orbits of $f \in \boldsymbol{R a t}_{d}^{\mu}$ avoid $\infty$. Let $\mathcal{F}$ be a finite set of critical relations $\left(c_{i_{k}}, c_{j_{k}}, m_{k}, n_{k}\right), k=1,2, \ldots, N$, which are realized by $f$ and which satisfies the non-cyclic condition (3.3). If the Jacobian of the map

$$
\boldsymbol{R a t}_{d}^{\boldsymbol{\mu}} \ni g \mapsto\left\{g^{m_{k}}\left(c_{i_{k}}(g)\right)-g^{n_{k}}\left(c_{j_{k}}(g)\right)\right\}_{k=1}^{N}
$$

at $g=f$ has rank less than $N$, then there exist complex numbers $a_{1}, a_{2}, \cdots, a_{N}$, such that

- for some $k,\left(m_{k}, n_{k}\right) \neq(1,1)$ and $a_{k} \neq 0$;

- $f_{*}\left(q(z) d z^{2}\right)=q(z) d z^{2}$, where

$$
q(z)=\sum_{\substack{1 \leq k \leq N \\\left(m_{k}, n_{k}\right) \neq(1,1)}} a_{k} Q_{i_{k}, j_{k} ; m_{k}, n_{k}}^{f}(z)
$$

If in addition $f$ is not a flexible Lattés example, then $q(z) \equiv 0$.

Remark 3.7 If $f$ has $2 d-2$ distinct critical values, then the converse statement of the theorem also holds. Namely, for any finite set $\mathcal{F}$ as above, if $f_{*}\left(q(z) d z^{2}\right)=q(z) d z^{2}$, then the Jacobian of the map (3.1) has rank less than $N$.

Remark 3.8 Take $f \in \mathbf{R a t}_{d}^{\mu}$ and a manifold $S$ passing through $f$ of dimension $p$, that is transverse to the orbit $O(f)$ of $f$ under Möbius conjugacies. Assume that the map defined in (3.1) has maximal rank. Then the restriction of this map to $S$ also has maximal rank. This holds because the value of the map (3.1) is constant on $O(f)$. 


\section{Theorems 3.1-3.2 Follow from Theorem 3.3}

\subsection{Proof of Theorem 3.1}

If $f^{m}\left(c_{i}\right)=c_{j}$ then $f$ is not a Lattés example. We may assume without loss of generality that $\infty$ avoids the critical orbits of $f$ so that Theorem 3.3 applies. It is clear that

$$
Q_{i, j ; m, 0}^{f}(z)=\frac{D f^{m-1}\left(f\left(c_{i}\right)\right)}{z-f\left(c_{i}\right)}+\cdots+\frac{1}{z-f^{m}\left(c_{i}\right)}
$$

has a pole at $c_{j}$, so it is not identically zero and thus the conclusion follows from the last sentence of Theorem 3.3.

\subsection{An Improved Way to Organise Critical Relations and the Proof of Theorem 3.2}

In general, one can associate several full collections $\mathcal{F}$ to $f$ each giving rise to a map $\mathcal{R}_{\mathcal{F}}^{\sigma}$ as in (3.1). Let us first prove, as claimed in Remark 3.2, that any full collection gives rise to the same rank:

Lemma 4.1 For any full collections $\mathcal{F}$ and $\mathcal{F}^{\prime}$ of critical relations for $f$, the Jacobian matrices of $\mathcal{R}_{\mathcal{F}}^{\sigma}$ and $\mathcal{R}_{\mathcal{F}^{\prime}}^{\sigma}$ at $g=f$ have the same rank.

Proof According to Remark 3.1, we may assume the critical orbits avoid $\infty$ and $\sigma=i d$. Consider a holomorphic curve $f_{t}$, passing through $f$ at $t=0$. This curve represents a vector in the kernel of $D \mathcal{R}_{\mathcal{F}}$ if and only if the derivative of $t \mapsto$ $f_{t}^{m}\left(c_{i}\left(f_{t}\right)\right)-f_{t}^{n}\left(c_{j}\left(f_{t}\right)\right)$ vanishes at $t=0$ for each $(i, j ; m, n) \in \mathcal{F}$, and therefore if and only if $t \mapsto f_{t}^{m}\left(c_{i}\left(f_{t}\right)\right)-f_{t}^{n}\left(c_{j}\left(f_{t}\right)\right)$ vanishes at $t=0$ for each $(i, m) \sim_{\mathcal{F}}(j, n)$ where $\sim_{\mathcal{F}}$ is the equivalence relation associated to $\mathcal{F}$ as defined in the first paragraph of Sect. 3.1.

Assume that $(i, j ; m, n)$ is realised by $f$. Since $\mathcal{F}$ is full, there exists $k \geq$ 0 so that $(i, m+k) \sim_{\mathcal{F}}(j, n+k)$ and so that $D f^{k}\left(f^{m}\left(c_{i}\right)\right)=D f^{k}\left(f^{n}\left(c_{j}\right)\right) \neq$ 0 . So if $f_{t}$ represents a vector in the kernel of $D \mathcal{R}_{\mathcal{F}}$ then the derivative of $t \mapsto f_{t}^{m+k}\left(c_{i}\left(f_{t}\right)\right)-f_{t}^{n+k}\left(c_{j}\left(f_{t}\right)\right)$ vanishes at $t=0$. Since $f^{m}\left(c_{i}\right)=f^{n}\left(c_{j}\right)$ and $D f^{k}\left(f^{m}\left(c_{i}\right)\right)=D f^{k}\left(f^{n}\left(c_{j}\right)\right) \neq 0$, this implies that the derivative of $t \mapsto f_{t}^{m}$ $\left(c_{i}\left(f_{t}\right)\right)-f_{t}^{n}\left(c_{j}\left(f_{t}\right)\right)$ vanishes at $t=0$.

On the other hand, if for each $(i, j ; m, n)$ which is realised by $f$ the derivative of $t \mapsto f_{t}^{m}\left(c_{i}\left(f_{t}\right)\right)-f_{t}^{n}\left(c_{j}\left(f_{t}\right)\right)$ vanishes at $t=0$, then in particular this holds for each $(i, j ; m, n) \in \mathcal{F}$ and so the holomorphic curve $f_{t}$ represents a vector in the kernel of $D \mathcal{R}_{\mathcal{F}}$.

It follows that $f_{t}$ represents a vector in the kernel of $D \mathcal{R}_{\mathcal{F}}$ if and only if for each $(i, j ; m, n)$ which is realised by $f$ the derivative of $t \mapsto f_{t}^{m}\left(c_{i}\left(f_{t}\right)\right)-f_{t}^{n}\left(c_{j}\left(f_{t}\right)\right)$ vanishes at $t=0$. The last condition is independent of the choice of the full collection 
$\mathcal{F}$. Since both $\mathcal{F}$ and $\mathcal{F}^{\prime}$ are full, the rank-nullity theorem implies that the rank of the Jacobian matrices are the same.

We will find it convenient to prove Theorem 3.2 for a conveniently chosen minimal collection $\mathcal{F}$, namely one which satisfies the following stronger minimality assumption.

Definition 4.2 We say that a collection $\mathcal{F}$ is proper for $f$ if it is minimally full and satisfies the following extra properties:

(1) $(i, j ; m, n) \in \mathcal{F}$ implies $m>0$ and either $i \geq j$ or $n=0$. If $i=j$ then $m>n$.

(2) if $(i, j ; m, n) \in \mathcal{F}$ then the collection of points $f^{k}\left(c_{i}\right), k=1, \ldots, m-1$ is pairwise disjoint and does not intersect $c_{1}, \ldots, c_{v}$ nor the forward orbits of $c_{1}, \ldots, c_{i-1}$.

(3) For each $1 \leq i \leq v$ there exists at most one critical relation of the form $(i, j ; m, n) \in \mathcal{F}$.

(4) For each $1 \leq j \leq v$ there exists at most one critical relation of the form $(i, j ; m, 0) \in \mathcal{F}$

(5) For each $1 \leq j \leq v$ and each $n>1$ there exists at most one critical relation of the form $(i, j ; 1, n) \in \mathcal{F}$;

(6) If $(i, j ; m, n) \in \mathcal{F}$ with $m>1$ and $n>0$, and $(k, i ; 1, l) \in \mathcal{F}$ for some $k$ and $l$, then $l<m$.

Remark 4.3 If the collection $\mathcal{F}$ is proper then it satisfies the non-cyclic condition (3.3).

Lemma 4.4 There exists a proper collection of critical relations which are realised by $f$.

Proof For each $i=1, \ldots, v$, inductively define $m_{i}>0$ maximal so that $f\left(c_{i}\right), \ldots$, $f^{m_{i}-1}\left(c_{i}\right)$ are distinct and also distinct from

$$
\left\{f^{k}\left(c_{j}\right) ; 0 \leq k<m_{j}, j=1, \ldots, i-1\right\} \cup\left\{c_{1}, \ldots, c_{\nu}\right\}
$$

(When $i=1$ we take this union to be $\left\{c_{1}, \ldots, c_{\nu}\right\}$.) If $m_{i}$ is finite, then there are two possibilities:

(a) $f^{m_{i}}\left(c_{i}\right)=f^{n_{j_{i}}}\left(c_{j_{i}}\right)$ for some $1 \leq j_{i} \leq i$ and some finite $n_{j_{i}}$ with $0<n_{j_{i}}<m_{j_{i}}$. In this case associate to $c_{i}$ the critical relation $\left(i, j_{i}, m_{i}, n_{j_{i}}\right)$.

(b) $f^{m_{i}}\left(c_{i}\right)=c_{j}$ with $1 \leq j \leq v$ and in this case associate to $c_{i}$ the critical relation $\left(i, j, n_{i}, 0\right)$.

These choices ensure that properties (1) and (2) in the above definition hold. To take care that properties (3)-(6) hold we also make the following requirement:

If both (a) and (b) hold, then only assign to $c_{i}$ the critical relation as in (a). If (a) holds for several $j_{i} \leq i$, then choose the smallest possible $j_{i}$ with $n_{j_{i}}=1$ and if there is no $j_{i}$ with $n_{j_{i}}=1$ then simply choose the smallest possible $j_{i}$. Assign to $i$ 
only the corresponding critical relation. Once we have done this for $i$ then repeat this construction for $i+1$.

In this way we define no new critical relation for each $1 \leq i \leq v$ whose orbit is infinite and disjoint from forward orbits of $c_{1}, \ldots, c_{i-1}$ and from $c_{1}, \ldots, c_{\nu}$, but a unique critical relation for each of the other $i$ 's. Thus we get $N=v-\zeta(f)$ critical relations.

The resulting set of critical relations is realised by $f$. By construction $\mathcal{F}$ is proper.

Proof of Theorem 3.2. By Remark 3.1, it is enough to consider the case that $\sigma$ is the identity and the critical orbits avoid $\infty$. Let $\mathcal{F}$ be a proper collection of critical relations realized by $f$. Note that if $m, n \geq 1$ then $Q_{i, k ; m, n}^{f}(z)$ is equal to

$$
Q_{i, j ; m, n}^{f}(z)=\sum_{r=1}^{m-1} \frac{D f^{m-r}\left(f^{r}\left(c_{i}\right)\right)}{z-f^{r}\left(c_{i}\right)}-\sum_{s=1}^{n-1} \frac{D f^{n-s}\left(f^{s}\left(c_{j}\right)\right)}{z-f^{s}\left(c_{j}\right)}
$$

and if $m=n=1$ then $Q_{i, j ; m, n}^{f}(z)=0$. By property (2) of Definition 4.2, $(i, j ; m, n)$ $\in \mathcal{F}$ and $m, n \geq 1$ imply

$$
D f^{m-1}\left(f\left(c_{i}\right)\right) \neq 0, D f^{n-1}\left(f\left(c_{j}\right)\right) \neq 0
$$

and if $i \neq j$ then

$$
f\left(c_{i}\right), \ldots, f^{m-1}\left(c_{i}\right), f\left(c_{j}\right), \ldots, f^{n-1}\left(c_{j}\right)
$$

are all distinct and distinct from $c_{1}, \ldots, c_{\nu}$. Similarly, if $i=j$ then by properties (1), (2) of Definition 4.2, $m>n$ and $f\left(c_{i}\right), \ldots, f^{m-1}\left(c_{i}\right), c_{1}, \ldots, c_{v}$ are all distinct. Hence, if $m, n \geq 1$ and $i \neq j$ then $Q_{i, j ; m, n}^{f}(z)$ has a non-removable pole in each of the points from the collection (4.3) and nowhere else. In particular, $c_{1}, \ldots, c_{v}$ is not a pole for any $Q_{i, j ; m, n}(z)$ when $m, n \geq 1$ (this holds even when $i=j$ ). On the other hand, $Q_{i, j ; m, 0}^{f}(z)$ does have a pole at $c_{j}$ and only critical relations of this form in $\mathcal{F}$ have a pole at $c_{j}$.

Suppose that the Jacobian does not have full rank. By Theorem 3.3 this implies

$$
\sum_{\substack{1 \leq k \leq N \\\left(m_{k}, n_{k}\right) \neq(1,1)}} a_{k} Q_{i_{k}, j_{k} ; m_{k}, n_{k}}^{f}(z)=0
$$

Let $\mathcal{F}_{0}$ be the set of relations $\left(i_{k}, j_{k} ; m_{k}, n_{k}\right)$ in $\mathcal{F}$ in this sum for which $a_{k} \neq 0$ and with $\left(m_{k}, n_{k}\right) \neq(1,1)$. So $(4.4)$ is equal to the sum over the set $\mathcal{F}_{0}$. By Theorem 3.3, $\mathcal{F}_{0}$ consists of at least one critical relation, and obviously the properties stated in Definition 4.2 are also satisfied for $\mathcal{F}_{0}$.

Suppose first that there exists a critical relation $(i, j ; m, 0) \in \mathcal{F}_{0}$. In this case by property (4) in Definition 4.2 there exists no $i^{\prime} \neq i, m^{\prime}>0$ so that $\left(i^{\prime}, j ; m^{\prime}, 0\right) \in \mathcal{F}_{0}$. 
It follows from this that $(i, j ; m, 0)$ is the only term in the sum (4.4) which leads to a pole at $z=c_{j}$. So the corresponding coefficient $a_{k}=0$, a contradiction.

From now on, let us assume that for any $(i, j ; m, n) \in \mathcal{F}_{0}, n>0$. Then by property (1) of Definition 4.2, we have $i \geq j$. Because of property (3) of Definition 4.2 we can rearrange, if necessary, the critical relations in $\mathcal{F}_{0}$ so that they are of the form $\left(i_{k}, j_{k} ; m_{k}, n_{k}\right), 1 \leq k \leq N_{0}$, with $i_{1}<i_{2}<\cdots<i_{N_{0}}$. If $m_{k}=1$ holds for all $1 \leq$ $k \leq N_{0}$, then by property (5), $\left(j_{k}, n_{k}\right)$ are pairwise distinct. Since $Q_{i_{k}, j_{k} ; 1, n_{k}}^{f}$ has poles precisely at the points $f\left(c_{j_{k}}\right), f^{2}\left(c_{j_{k}}\right), \cdots, f^{n_{k}-1}\left(c_{j_{k}}\right), \sum_{k=1}^{N_{0}} a_{k} Q_{i_{k}, j_{k} ; m_{k}, n_{k}}^{f}$ has a pole, a contradiction! So let us assume that there is a maximal $N_{1} \leq N_{0}$ such that $m_{N_{1}} \geq 2$. By property (6) of Definition 4.2, for each $N_{0} \geq k>N_{1}$, either $j_{k} \neq i_{N_{1}}$, or $j_{k}=i_{N_{1}}$ and $n_{k}<m_{N_{1}}$. Together with property (2) of Definition 4.2, this implies that $Q_{i_{k}, j_{k} ; m_{k}, n_{k}}^{f}=Q_{i_{k}, j_{k} ; 1, n_{k}}^{f}$ does not have a pole at $f^{m_{N_{1}}-1}\left(c_{i_{N_{1}}}\right)$. For each $k<N_{1}$, since $i_{N_{1}}>i_{k} \geq j_{k}$, by property (2) of Definition 4.2, $Q_{i_{k}, j_{k} ; m_{k}, n_{k}}^{f}$ does not have a pole at $f^{m_{N_{1}}-1}\left(c_{N_{1}}\right)$ either. Therefore $\sum_{k=1}^{N_{0}} a_{k} Q_{i_{k}, j_{k} ; m_{k}, n_{k}}^{f}$ has a pole at $f^{m_{N-1}-1}\left(c_{N_{1}}\right)$, a contradiction!

\section{$5 \quad$ A Proof of Theorem 3.3}

Remark 5.1 A proof of Theorem 3.3 is contained essentially in [20]. We only outline it here (and then present another proof). Denote $v_{j}(f)=f\left(c_{j}(f)\right)$ for $j=1, \cdots, v$. Conjugating $f$ by a Möbius transformation, one can assume that $f(\infty)=\infty$, $D f(\infty) \neq 0$. We label the critical values so that for some $0 \leq v^{\prime} \leq v$ the following holds: $v_{j}(f) \in \mathbb{C}$ for $1 \leq j \leq v^{\prime}$ and $v_{j}(f)=\infty$ for $v^{\prime}<j \leq v$. Consider a subset $\Lambda_{f, v} \subset$ Rat $_{d}^{\mu}$ of maps $g$ such that there exists $\sigma(g), b(g) \in \mathbb{C}$ so that $g(z)=\sigma(g) z+$ $b(g)+O(1 / z)$ as $z \rightarrow \infty$. By [19], $\Lambda_{f, v}$ has a structure of $v+2$ dimensional complex manifold and $\left(\sigma(g), b(g), v_{1}(g), \cdots, v_{v^{\prime}}(g), v_{v^{\prime}+1}(g)^{-1}, \cdots, v_{v}(g)^{-1}\right)$ is a holomorphic coordinate of $g \in \Lambda_{f, v}$. Proposition 10 of [20] implies that for any $(i, j ; m, n)$, if $(i, j ; m, n)$ is realized by $f$ and $f^{m}\left(c_{i}(f)\right), f^{n}\left(c_{j}(f)\right) \neq \infty$, then

$$
\begin{aligned}
Q_{i, j ; m, n}^{f}(x)- & \widehat{Q}_{i, j ; m, n}^{f}(x)= \\
& \left.\sum_{k=1}^{v^{\prime}} \frac{1}{v_{k}(f)-x} \frac{\partial\left(g^{m}\left(c_{i}(g)\right)-f^{n}\left(c_{j}(g)\right)\right)}{\partial v_{k}}\right|_{g=f},
\end{aligned}
$$

where $\widehat{Q}_{i, j ; m, n}^{f}(x) d x^{2}=f_{*}\left(Q_{i, j ; m, n}^{f}(x) d x^{2}\right)$. Now Theorem 3.3 can be proved by repeating the proof of the main result of [20] after replacing Proposition 13 of that paper by (5.1). Instead of going into more details we give here a direct and short proof of the theorem. 


\subsection{Proof of Theorem 3.3.}

Let us first apply Thurston's pull back argument to obtain a relation of partial derivatives of $g \mapsto g^{m}\left(c_{i}(g)\right)-g^{n}\left(c_{j}(g)\right)$ with the quadratic differential $Q_{i, j ; m, n}(z) d z^{2}$. Let $L_{\infty}(\mathbb{C})$ denote the space of all Borel measurable functions $\mu$ with $\|\mu\|_{\infty}<\infty$. Note that $f^{*} \mu(z)=\mu(f(z)) \overline{f^{\prime}(z)} / f^{\prime}(z)$ also belongs to the class $L_{\infty}(\mathbb{C})$.

Lemma 5.2 Given $\mu \in L_{\infty}(\mathbb{C})$ which vanishes in a neighborhood of $\infty$ and $f(\infty)$, there exists a holomorphic family $f_{t}$ of rational maps of degree $d, t \in \mathbb{D}_{\mathcal{E}}$, with $f_{0}=f$, and such that the following holds: For any $(i, j ; m, n)$,

$$
-\frac{1}{\pi} \int_{\mathbb{C}}\left(\mu-f^{*} \mu\right) Q_{i, j ; m, n}|d z|^{2}=\left.\frac{d\left(f_{t}^{m}\left(c_{i}\left(f_{t}\right)\right)-f_{t}^{n}\left(c_{j}\left(f_{t}\right)\right)\right)}{d t}\right|_{t=0} .
$$

Proof Assume without loss of generality that $\|\mu\|_{\infty} \leq 1$. Then for each $t \in \mathbb{D}$, there are qc maps $\varphi_{t}, \psi_{t}: \mathbb{C} \rightarrow \mathbb{C}$ with complex dilatations $t \mu$ and $t f^{*} \mu$ respectively such that (see e.g. [1])

- $\varphi_{t}(z)=z+o(1), \psi_{t}(z)=z+o(1)$ as $z \rightarrow \infty$ for each $t$

- $f_{t}$ defined by $f_{t} \circ \psi_{t}=\varphi_{t} \circ f$ is a family of rational maps.

Then $\varphi_{t}$ and $\psi_{t}$ depends on $t$ holomorphically [1] and thus $\partial f_{t} / \bar{\partial} t=0$ in the sense of distribution, which implies that $f_{t}$ depends holomorphically on $t$. Let

$$
\mathcal{L}_{n}(z)=\left.\frac{d f_{t}^{n}(z)}{d t}\right|_{t=0}
$$

and $L(z)=\mathcal{L}_{1}(z)$. Then

$$
L(x)+D f(z) \frac{d}{d t} \psi_{t}(z)=\frac{d}{d t} \phi_{t}(f(z))
$$

and therefore

$$
L(z)+D f(z) \widehat{X}(z)=X(f(z))
$$

where

$$
X(z)=-\frac{1}{\pi} \int_{\mathbb{C}} \frac{\mu(\zeta)}{\zeta-z}|d \zeta|^{2}, \widehat{X}(z)=-\frac{1}{\pi} \int_{\mathbb{C}} \frac{f^{*} \mu(\zeta)}{\zeta-z}|d \zeta|^{2}
$$

The latter formulas come from the following fact. Let $v \in L_{\infty}(\mathbb{C})$ have a compact support, $\|v\|_{\infty} \leq 1$ and $h_{t}(|t|<1)$ is the (unique) qc map with complex dilatation $t v$ such that $h_{t}(z)=z+o(1)$ as $z \rightarrow \infty$. Then

$$
\left.\frac{d h_{t}(z)}{d t}\right|_{t=0}=-\frac{1}{\pi} \int_{\mathbb{C}} \frac{v(\zeta)}{\zeta-z}|d \zeta|^{2} .
$$


The formula (5.4) is well-known and follows for example from the formula (6) in the proof of Theorem 1 of [1], Chap. V (noting that a different normalisation for $h_{t}$ is chosen there) or by differentiating the formula on the 2nd line of page 25 in [4].

For any $h \in\{1,2, \ldots, v\}$ and non-negative integer $l$, define

$$
S_{l}\left(c_{h}\right)=\sum_{r=1}^{l} D f^{l-r}\left(f^{r}\left(c_{h}\right)\right) X\left(f^{r}\left(c_{h}\right)\right)
$$

and

$$
\widehat{S}_{l}\left(c_{h}\right)=\sum_{r=1}^{l} D f^{l-r}\left(f^{r}\left(c_{h}\right)\right) \widehat{X}\left(f^{r}\left(c_{h}\right)\right)
$$

It suffices to show that for any $l, h$ as above,

$$
S_{l}\left(c_{h}\right)-\widehat{S}_{l}\left(c_{h}\right)=\left.\frac{d f_{t}^{l}\left(c_{h}\left(f_{t}\right)\right)}{d t}\right|_{t=0}-\widehat{X}\left(f^{l}\left(c_{h}\right)\right) .
$$

If $l=0$, then the left hand is equal to zero, and the right hand side is also equal to zero, since $\widehat{X}\left(c_{h}\right)=\left.\frac{d \psi_{t}\left(c_{h}\right)}{d t}\right|_{t=0}$ and $c_{h}\left(f_{t}\right)=\psi_{t}\left(c_{h}\right)$. For $l \geq 1$, we use (5.3):

$$
\begin{aligned}
S_{l}\left(c_{h}\right) & =\sum_{r=1}^{l} D f^{l-r}\left(f^{r}\left(c_{h}\right)\right) X\left(f^{r}\left(c_{h}\right)\right) \\
& =\sum_{r=1}^{l} D f^{l-r}\left(f^{r}\left(c_{h}\right)\right) L\left(f^{r-1}\left(c_{h}\right)\right)+\sum_{r=2}^{l} D f^{l-r+1}\left(f^{r-1}\left(c_{h}\right)\right) \widehat{X}\left(f^{r-1}\left(c_{h}\right)\right) \\
& =\mathcal{L}_{l}\left(c_{h}\right)+\sum_{r=1}^{l-1} D f^{l-r}\left(f^{r}\left(c_{h}\right)\right) \widehat{X}\left(f^{r}\left(c_{h}\right)\right) \\
& =\mathcal{L}_{l}\left(c_{h}\right)+\widehat{S}_{l}\left(c_{h}\right)-\widehat{X}\left(f^{l}\left(c_{h}\right)\right) .
\end{aligned}
$$

Since

$$
\left.\frac{d f_{t}^{l}\left(c_{h}\left(f_{t}\right)\right)}{d t}\right|_{t=0}=\left.\frac{d f_{t}^{l}\left(c_{h}\right)}{d t}\right|_{t=0}
$$

Equation (5.5) follows.

Proof (Proof of Theorem 3.3) Assume that the Jacobian matrix has rank less than $N$. Then there exist complex numbers $a_{1}, a_{2}, \cdots, a_{N}$ such that all the partial derivatives of the map

$$
g \mapsto \sum_{k=1}^{N} a_{k}\left(g^{m_{k}}\left(c_{i_{k}}(g)\right)-g^{n_{k}}\left(c_{j_{k}}(g)\right)\right)
$$


is equal to 0 at $g=f$. Since $\mathcal{F}$ satisfies the non-cyclic condition (3.3), by Theorem 2.1, there exists $k$ such that $\left(m_{k}, n_{k}\right) \neq(1,1)$ and $a_{k} \neq 0$.

Given $\mu \in L_{\infty}(\mathbb{C})$ which vanishes in a neighbourhood of $\infty$ and $f(\infty)$, let $f_{t}$ be given by the previous lemma. Then for each $k=1,2, \ldots, N$, we have

$$
-\frac{1}{\pi} \int_{\mathbb{C}}\left(\mu-f^{*} \mu\right) Q_{i_{k}, j_{k} ; m_{k}, n_{k}}|d z|^{2}=\left.\frac{d\left(f_{t}^{m}\left(c_{i}\left(f_{t}\right)\right)-f_{t}^{n}\left(c_{j}\left(f_{t}\right)\right)\right)}{d t}\right|_{t=0} .
$$

Thus for $q$ defined as in (3.5), and $\widehat{q}(z) d z^{2}=f_{*}\left(q(z) d z^{2}\right)$, we have

$$
\begin{aligned}
& \int_{\mathbb{C}} \mu(\hat{q}-q)|d z|^{2} \\
= & \int_{\mathbb{C}}\left(\mu-f^{*} \mu\right) q(z)|d z|^{2} \\
= & -\left.\pi \sum_{k=1}^{N} a_{k} \frac{d\left(f_{t}^{m}\left(c_{i}\left(f_{t}\right)\right)-f_{t}^{n}\left(c_{j}\left(f_{t}\right)\right)\right)}{d t}\right|_{t=0}=0,
\end{aligned}
$$

where the last equality follows from the argument in the previous paragraph. It follows that $\widehat{q}=q$.

Assume now that $f$ is not a flexible Lattés example. Let us prove that $q=0$. To this end, first assume $f(\infty) \neq \infty$. Let $\varphi_{i}$ be the local inverse diffeomorphic branches of $f$ near $\infty$. Then $\hat{q}=q$ implies that

$$
q(z)=\sum_{i=1}^{d} q\left(\varphi_{i}(z)\right) \varphi_{i}^{\prime}(z)^{2}
$$

holds near $\infty$. Since $\varphi_{i}(\infty) \in \mathbb{C}$ (and is not equal to one of the finitely many poles of $q)$ and $\varphi_{i}^{\prime}(z)=O\left(1 / z^{2}\right)$ as $z \rightarrow \infty$, it follows from the displayed formula $q(z)=$ $O\left(1 / z^{4}\right)$ at infinity. Thus $q(z) d z^{2}$ is an integrable meromorphic quadratic differential. By a well-known argument, this implies that $q(z)=0$, see for example Sect. 3.5 of $[6,24]$.

If $f(\infty)=\infty$, then we can find a sequence of Möbius transformations $\sigma_{l}, l=$ $1,2, \ldots$, converging to the identity uniformly, such that $f_{(l)}=\sigma_{l} \circ f \circ \sigma_{l}^{-1}$ satisfies $f_{(l)}(\infty) \neq \infty$ and $\infty$ avoids the critical orbits of $f_{(l)}$. Putting $g_{(l)}=\sigma_{l} \circ g \circ \sigma_{l}^{-1}$, by (5.6), all partial derivatives of the map

$$
g \mapsto \sum_{k=1}^{N} a_{k}\left(\sigma_{l}^{-1}\left(g_{(l)}^{m_{k}}\left(c_{i_{k}}\left(g_{(l)}\right)\right)\right)-\sigma_{l}^{-1}\left(g_{(l)}^{n_{k}}\left(c_{j_{k}}\left(g_{(l)}\right)\right)\right)\right)
$$

are equal to zero at $g=f$, hence all partial derivatives of the map 


$$
g \mapsto \sum_{k=1}^{N} \frac{a_{k}}{\sigma_{(l)}^{\prime}\left(f^{m_{k}}\left(c_{i_{k}}\right)\right)}\left(\left(g^{m_{k}}\left(c_{i_{k}}(g)\right)-g^{n_{k}}\left(c_{j_{k}}(g)\right)\right)\right.
$$

are equal to zero at $g=f_{(l)}$. Since $f_{(k)}$ is not a Lattés example, as above we obtain that $q_{(l)}:=\sum_{k=1}^{N} \frac{a_{k}}{\sigma_{(l)}^{\prime}\left(f^{m_{k}}\left(c_{i_{k}}(f)\right)\right)} Q_{i_{k}, j_{k} ; m_{k}, n_{k}}^{f_{(l)}} \equiv 0$. By continuity we conclude that $q=0$.

\section{The Polynomial Case}

The previous theorems also hold in the space of polynomials of degree $d$. In that case, let $\boldsymbol{\mu}=\left(\mu_{1}, \ldots, \mu_{v}\right)$ so that $\sum_{i=1}^{v} \mu_{i}=d-1$ and let Pol $_{d}^{\boldsymbol{\mu}}$ be the set of maps with critical points $c_{1}, \ldots, c_{v} \in \mathbb{C}$ of orders $\mu_{1}, \ldots, \mu_{v}$. The space $\mathbf{P o l}_{d}^{\mu}$ is clearly an embedded submanifold of $\mathbf{R a t}_{d}^{\hat{\mu}}$ of codimension one, where $\hat{\boldsymbol{\mu}}=\left(\mu_{1}, \mu_{2}, \cdots, \mu_{v}, d-1\right)$.

Theorem 6.1 Assume $f \in$ Pol $_{d}^{\mu}$. Then there exists a set

$$
\mathcal{F}=\left\{\left(i_{k}, j_{k} ; m_{k}, n_{k}\right), k=1, \ldots, N\right\} \text { with } N=v-\zeta(f)
$$

of critical relations $f^{m_{k}}\left(c_{i_{k}}\right)=f^{n_{k}}\left(c_{j_{k}}\right)$ which are realised by $f$, such that the Jacobian of the map

$$
\boldsymbol{P o l}_{d}^{\mu} \ni g \mapsto\left(g^{m_{k}}\left(c_{i_{k}}(g)\right)-g^{n_{k}}\left(c_{j_{k}}(g)\right)\right)_{k=1}^{N}
$$

at $g=f$ has rank $N$.

Proof Let $c_{\nu+1}=\infty$. For maps $g$ in $\mathbf{R a t}_{d}^{\hat{\mu}}$ close to $f$, let $c_{j}(g)$ denote the critical point of $g$ close to $c_{i}, 1 \leq i \leq v+1$. By Theorem 3.2, there is a set $\widehat{\mathcal{F}}=$ $\left\{\left(i_{k}, j_{k} ; m_{k}, n_{k}\right)\right\}_{j=1}^{N+1}$ of critical relations of $f$ so that the Jacobian of the map

$$
\mathcal{R}_{\widehat{\mathcal{F}}}^{\sigma}: \boldsymbol{R a t}_{d}^{\hat{\mu}} \ni g \mapsto\left(\sigma\left(g^{m_{k}}\left(c_{i_{k}}(g)\right)\right)-\sigma\left(g^{n_{k}}\left(c_{j_{k}}(g)\right)\right)\right)_{k=1}^{N+1}
$$

has rank $N+1$ at $g=f$, where $\sigma$ is a Möbius tansformation such that $\sigma\left(f^{m_{k}}\left(c_{i_{k}}\right)\right) \neq$ $\infty$ for all $k$. Since $\widehat{\mathcal{F}}$ is full, there is $k_{0}$ such that $\left(i_{k_{0}}, j_{k_{0}} ; m_{k_{0}}, n_{k_{0}}\right)=(v+1, v+$ $1 ; 1,0)($ or $(v+1, v+1 ; 0,1))$. Assume without loss of generality $k_{0}=N+1$. Let $\mathcal{F}=\left\{\left(i_{k}, j_{k} ; m_{k}, n_{k}\right): 1 \leq k \leq v\right\}$ and let $\mathcal{R}$ denote the map defined by (6.1). Note that the kernel of $D_{f} \mathcal{R}$ is contained in the kernel of $D_{f} \mathcal{R}_{\widehat{\mathcal{F}}}^{\sigma}$, so its dimension is at $\operatorname{most} \operatorname{dim}\left(\boldsymbol{R a t}_{d}^{\hat{\mu}}\right)-(N+1)=\operatorname{dim}\left(\mathbf{P o l}_{d}^{\boldsymbol{\mu}}\right)-N$. Thus the rank of $D_{f} \mathcal{R}$ is at least $N$. The rank is not more than $N$, so it is equal to $N$. 
Acknowledgements The authors thank Alex Eremenko for a discussion about Theorem 2.1, Adam Epstein for pointing out an error in the last paragraph of Sect. 5.1 in a previous version, Xavier Buff and Lasse Rempe-Gillen for helpful comments on the introduction of the final version of this paper and the referee for carefully reading the paper. This project was partly supported by the ISF grant no: 1226/17, ERC AdG grant no: 339523 RGDD and the NSFC grant no: 11731003 .

\section{References}

1. L.V. Ahlfors, Lectures on quasiconformal mappings, vol. 38, 2nd edn. Van Nostrand, Princeton 1966; A.M.S. University Lecture Series (2006)

2. M. Astorg, Summability condition and rigidity for finite type maps, arXiv:1602.05172v1

3. X. Buff, A. Epstein, Bifurcation measure and postcritically finite rational maps. Complex Dyn. 491, 512 (2009)

4. L. Carleson, T.W. Gamelin, Complex Dynamics (Springer, 1992)

5. A. Douady, J.H. Hubbard, Étude dynamique des polynômes complexes. Publications Mathématiques d'Orsay, 84-2. Université de Paris-Sud, Département de Mathématiques, Orsay (1984), $75 \mathrm{pp}$

6. A. Douady, J.H. Hubbard, A proof of Thurston's topological characterization of rational functions. Acta Math. 171(2), 263-297 (1993)

7. A. Epstein, Infinitesmimal Thurston rigidity and the Fatou-Shishikura inequality, Stony Brook IMS preprint 1999\#1

8. A. Epstein, Transversality in holomorphic dynamics, http://homepages.warwick.ac.uk/ mases/ Transversality.pdf

9. A. Epstein, Slides of talk available in https://icerm.brown.edu/materials/Slides/sp-s12-w1/ Transversality_Principles_in_Holomorphic_Dynamics_\%5D_Adam_Epstein,_University_ of_Warwick.pdf

10. A. Eremenko, A. Gabrielov, Rational functions with real critical points and the B. and M. Shapiro conjecture in real enumerative geometry. Ann Math. 155, 105-129 (2002)

11. A. Eremenko, M. Lyubich, Dynamical properties of some classes of entire functions. Ann. Inst. Fourier 42(4), 1-32 (1992)

12. A. Eremenko, A Markov-type inequality for arbitrary plane continua. Proc. AMS 135, 15051510 (2007)

13. C. Favre, T. Gauthier, Distribution of postcritically finite polynomials. Isr. J. Math. 209, 235292 (2015)

14. F. Gardiner, Teichmuller theory and quadratic differentials (Wiley, 1987)

15. G. Levin, M.L. Sodin, P.M. Yuditski, A Ruelle operator for a real Julia set. Comm. Math. Phys. 141(1), 119-132 (1991)

16. G.M. Levin, On the theory of iterations of polynomial families in the complex plane. Translation from: Toeriya Funkzii, Funkzionalnyi Analiz i Ih Prilozheniya, no. 51, 94-106 (1989)

17. G.M. Levin, Polynomial Julia sets and Pade's approximations (in Russian), in Proceedings of XIII Workshop on Operator's Theory in Functional Spaces, Kyubishev, 6-13 October 1988 (Kyubishev State University, Kyubishev, 1988), pp. 113-114

18. G. Levin, On an analytic approach to the Fatou conjecture. Fund. Math. 171(2), 177-196 (2002)

19. G. Levin, Multipliers of periodic orbits in spaces of rational maps. Ergod. Theory Dynam. Syst. 31, 197-243 (2011)

20. G. Levin, Perturbations of weakly expanding critical orbits. Front. Complex Dyn., 163-196 (2014). Princeton Math. Ser., 51, Princeton University Press, Princeton, NJ

21. G. Levin, W. Shen, S. van Strien, Monotonicity of entropy for one-parameter families of interval maps. Preprint Oct 2016

22. P. Makienko, Remarks on the Ruelle operator and the invariant line fields problem. II. Ergod. Theory Dynam. Syst. 25(5), 1561-1581 (2005) 
23. C. McMullen, Amenability, Poincaré series and quasiconformal maps. Invent. Math 97, 95-127 (1989)

24. C. McMullen, Complex Renormalisation and Renormalisation (Princeton University Press, Princeton, 1994)

25. W. de Melo, S. van Strien, One-dimensional dynamics, Ergebnisse der Mathematik und ihrer Grenzgebiete (Springer, Berlin, 1993)

26. J. Milnor, Tsujii's monotonicity proof for real quadratic maps, unpublished 2000

27. J. Milnor, On Lattès maps, in Dynamics on the Riemann Sphere, pp. $9-43$ (2006). Eur. Math. Soc, Zürich

28. J. Milnor, W. Thurston, On iterated maps of the interval, in Dynamical Systems, College Park, MD, 1986-87. Lecture Notes in Math., 1342 (Springer, Berlin, 1988), pp. 465-563

29. J. Milnor, C. Tresser, On entropy and monotonicity for real cubic maps. Commun. Math. Phys. 209, 123-178 (2000)

30. S. van Strien, Misiurewicz maps unfold generically (even if they are critically non-finite). Fund. Math. 163(1), 39-54 (2000)

31. O. Teichmüller, Eine Anwendung, quasikonformer Abbildungen auf das Typenproblem. Deutsche Math. 2, 321-327 (1937). Gesammelte Abhandlungen, Springer, Berlin, 1982, 171177

32. M. Tsujii, A note on Milnor and Thurston's monotonicity theorem, in Geometry and Analysis in Dynamical Systems (Kyoto, 1993), pp. 60-62, Adv. Ser. Dynam. Systems, 14, World Sci. Publ., River Edge, NJ, 1994

33. M. Tsujii, A simple proof of monotonicity of entropy in the quadratic family. Ergod. Theory Dynam. Syst. 20, 925-933 (2000) 